\title{
Interplay between steps and oxygen vacancies on curved $\mathrm{TiO}_{2}(110)$
}

\author{
Luis A. Miccio ${ }^{1,2^{*}}$, Martin Setvin ${ }^{3}$, Moritz Müller ${ }^{4}$, Mikel Abadía ${ }^{1}$, Ignacio Piquero ${ }^{1}$, Jorge \\ Lobo-Checa ${ }^{1}$, Frederik Schiller ${ }^{1}$, Celia Rogero ${ }^{1}$, Michael Schmid ${ }^{3}$, Daniel Sánchez-Portal ${ }^{1,2}$, \\ Ulrike Diebold $^{3}$, J. Enrique Ortega ${ }^{1,2,5^{*}}$. \\ ${ }^{1}$ Centro de Física de Materiales (CSIC-UPV/EHU), Manuel Lardizabal 5, 20018 San Sebastián, Spain \\ ${ }^{2}$ Donostia International Physics Center (DIPC) 20018 San Sebastián, Spain \\ ${ }^{3}$ Institute of Applied Physics, Vienna University of Technology, Wiedner Hauptstrasse 8-10/134, 1040 Vienna, \\ Austria \\ ${ }^{4}$ CIC nanoGUNE, Ave. Tolosa 76, 20018 San Sebastián, Spain \\ ${ }^{5}$ Departamento de Física Aplicada, Universidad del País Vasco (UPV/EHU), 20080 San Sebastián, Spain \\ luisalejandro_miccio@ehu.es, enrique.ortega@ehu.es \\ *Corresponding authors
}

\begin{abstract}
A vicinal rutile $\mathrm{TiO}_{2}(110)$ crystal with a smooth variation of atomic steps parallel to the [1-10] direction was analyzed locally with STM and ARPES. The step edge morphology changes across the samples, from [1-11] zig-zag faceting to straight [1-10] steps. A step-bunching phase is attributed to an optimal (110) terrace width, where all bridge-bonded $\mathrm{O}$ atom vacancies $\left(\mathrm{O}_{\mathrm{br}}\right.$ vac's) vanish. The [1-10] steps terminate with a pair of two-fold coordinated $\mathrm{O}$ atoms, which give rise to bright, triangular protrusions $\left(\mathrm{S}_{\mathrm{t}}\right)$ in STM. The intensity of the Ti 3d-derived gap state correlates with the sum of $\mathrm{O}_{\mathrm{br}}$ vac's plus $\mathrm{S}_{\mathrm{t}}$ protrusions at steps, suggesting that both $\mathrm{O}_{\mathrm{br}}$ vac's and steps contribute a similar effective charge to sample doping. The binding energy of the gap state shifts when going from the flat (110) surface toward densely stepped planes, pointing to differences in the $\mathrm{Ti}^{3+}$ polaron near steps and at terraces.
\end{abstract}

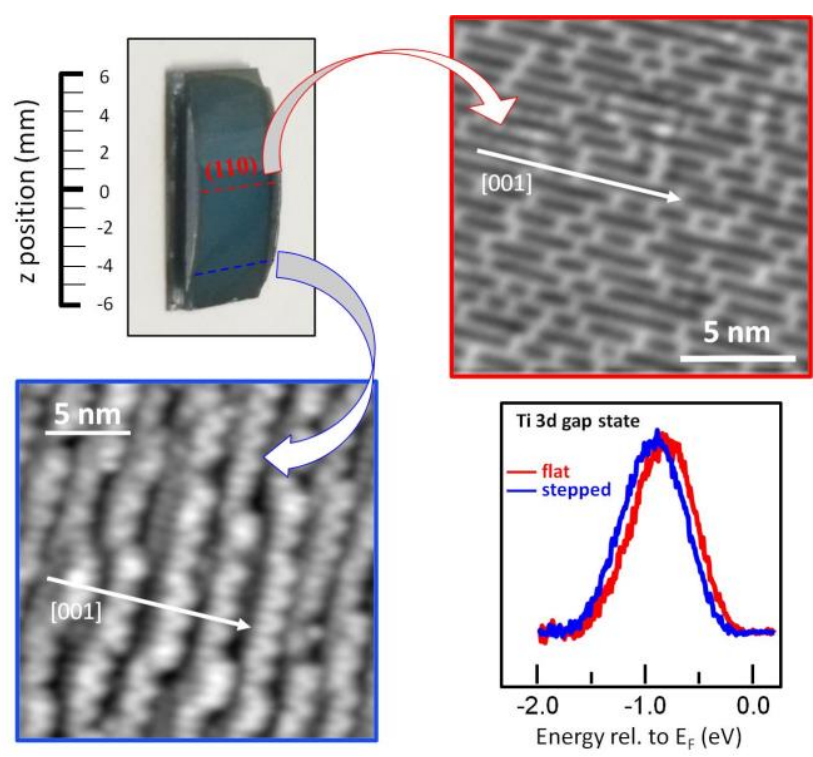


Rutile, the most stable polymorph of titanium dioxide $\left(\mathrm{r}-\mathrm{TiO}_{2}\right)$, is a prototypical, reducible metal oxide. It is applied in many fields ranging from catalysis to memristors ${ }^{1}$. These applications are often enabled by lattice defects, which act as electron donors and convert the otherwise insulating system into an n-type semiconductor. The nature of these defects, as well as their influence on the electronic and chemical behavior of reduced crystals $\left(\mathrm{r}-\mathrm{TiO}_{2}\right)$, has attracted considerable interest ${ }^{1-7}$. Of particular importance are oxygen vacancies, which donate electrons to the material. Excess electrons in rutile tend to localize at surface and subsurface Ti atoms, forming a $\mathrm{Ti}^{3+}$ oxidation state. ${ }^{5-9}$ The localized electron is accompanied by lattice distortions and can be characterized as a small polaron in $\mathrm{TiO}_{2}$ rutile. ${ }^{10}$

Step edges are sites with low atomic coordination and thus chemically and electronically active. Only a few studies ${ }^{11-15}$ on vicinal rutile planes exist, but they reveal the potential of stepped surfaces to further tailor the physical and chemical properties of $\mathrm{r}-\mathrm{TiO}_{2}$. Martinez et al. ${ }^{13}$ have reported the existence of oxygen vacancies at [1-11] step edges $\left(\mathrm{O}_{\mathrm{s}} \mathrm{vac}^{\prime} \mathrm{s}\right)$, as these are easier to form than $\mathrm{O}_{\mathrm{br}}$ vac's at terraces. For [1-10] steps Lutrell et al ${ }^{12}$ have proposed an atomic model without missing oxygen atoms (i.e., no $\mathrm{O}_{\mathrm{s}} v_{\mathrm{ac}}$ 's) that still explains the bright features they observe by Scanning Tunneling Microscopy (STM). A systematic study of different vicinal planes that explores the structural stability of both, the vicinal surface orientation and the step edge termination ${ }^{14}$, the existence of any characteristic step contributions to electron-doping ${ }^{13}$, or the possible alteration of the $\mathrm{Ti}^{3+}$ polaron in the vicinity of steps, is still missing.

In this Letter we demonstrate how a thorough exploration of structural and electronic properties of stepped $\mathrm{r}-\mathrm{TiO}_{2}(110)$ surfaces can be conveniently performed using curved crystals as samples. Our curved $\mathrm{TiO}_{2}(110)$ surface ${ }^{16}\left(\mathrm{c}-\mathrm{TiO}_{2}(110)\right)$ allows a systematic study of stepped $\mathrm{r}-$ $\mathrm{TiO}_{2}(110)$ surfaces with a tunable density of [1-10]-oriented steps. Using STM we quantitatively analyze the structure and distribution of steps and vacancies as a function of the average deviation (miscut $\alpha$ ) from the (110) surface. The surface smoothly evolves from wide terraces, containing $\mathrm{O}_{\mathrm{br}} \mathrm{vac}^{\prime}$ s, toward narrow (110) terraces, depleted of $\mathrm{O}_{\mathrm{br}} \mathrm{vac}$ 's, but with a high density of triangularly-shaped protrusions at steps $\left(\mathrm{S}_{\mathrm{t}}\right)$. Such $\mathrm{S}_{\mathrm{t}}-$ featured steps act as preferred dissociation sites for $\mathrm{H}_{2} \mathrm{O}$ molecules. Interestingly we find an $\mathrm{O}_{\mathrm{br}}$ vac's-free phase with a characteristic step spacing $d_{c}$, suggesting an energetically favorable, vicinal surface orientation. By scanning the photoemission beam in angle resolved photoemission spectroscopy (ARPES) across the curved sample, we study the spectroscopic signature of the $\mathrm{Ti}^{3+}$ polaron, i.e., Ti 3d-derived peak in the band gap. The Ti $3 \mathrm{~d}$ gap state reflects the transition from oxygenbridge to step-edge-doping across the curved surface through a $110 \mathrm{meV}$ shift in apparent binding energy, which in turn suggests differences in the polaronic relaxation for the two types 
of trapped electrons. The densities of $\mathrm{S}_{\mathrm{t}}$ protrusions and $\mathrm{O}_{\mathrm{br}}$ vac's correlates quantitatively with the gap state intensity, indicating that both triangular features at step edges and $\mathrm{O}_{\mathrm{br}} \mathrm{vac}^{\prime} \mathrm{s}$ at terraces contribute to the crystal doping equally. Density functional theory (DFT) calculations indicate that the electron doping is not an intrinsic property of the step-edge, but stems from the presence of $\mathrm{O}_{\mathrm{br}} \mathrm{vac}$ 's. We predict that $\mathrm{S}_{\mathrm{t}}$ protrusions of [1-10] step edges are favorable sites for the formation of $\mathrm{O}_{\mathrm{br}}$ vac's in $\mathrm{r}-\mathrm{TiO}_{2}(110)$.

STM measurements were performed on an Omicron LT-STM head at T $=80 \mathrm{~K}$. Tunneling currents $I_{t} \leq 0.1 \mathrm{nA}$ and positive sample bias voltages $\left(V_{S}\right.$, imaging empty states) ranging from $0.7 \mathrm{~V}$ to $1.8 \mathrm{~V}$ were used. ARPES measurements were performed at a different setup equipped with a hemispherical analyzer (Phoibos150) and a monochromatized Helium I ( $\mathrm{h} v=21.2 \mathrm{eV}$ ) source, with an overall energy/angle resolution of $40 \mathrm{meV} / 0.1^{\circ}$. All experiments were done using a rutile single crystal curved around the [110] symmetry direction (BihurCrystal Ltd. ${ }^{16}$ ), as schematically depicted in Fig. 1 (a). The curving process leads to a smooth $\alpha= \pm 12^{\circ}$ miscut variation around the (110) surface, i.e., a variable density $1 / d$ ( $d$ being the step spacing) of monatomic $(h=3.25 \AA, d=h / \sin \alpha)$ steps oriented parallel to the [1-10] direction.

Argon sputtering (1 keV) and annealing (up to1000 K) cycles both cleaned and reduced the c$\mathrm{TiO}_{2}(110)$ crystal surface. Sputtering was performed parallel to the steps, which prevents damaging the stepped regions. The selected annealing temperature was sufficient to heal the sputter-damage. We roughly define three different levels of reduction, judged from the characteristic color of the sample ${ }^{17}$. Starting from a transparent crystal, a sufficiently clean and conducting surface was achieved with $\sim 5$ sputtering-annealing cycles. This defines the slightly reduced sample, characterized by a faint blue color. The intermediate (blue crystal) and the strongly reduced (dark crystal) stage were reached with 30 and 60 sputtering-annealing cycles, respectively. The characteristic $1 \times 1$ Low Energy Electron Diffraction (LEED) pattern for the unreconstructed sample is shown in Fig. 1 (b). Further sputter-annealing beyond 100 cycles led to a $2 \times 1$ surface reconstruction ${ }^{18}$. With LEED we measured the step spacing at each sample position, which determines the local crystallographic plane. STM images were quantitatively analyzed through an automated routine, which rendered the average terrace width $\tilde{d}$ and its standard deviation $\sigma$ (see supplementary information $(\mathrm{SI})^{16}$ ) at each sample position. All STM images presented here are high-pass filtered, which enhances the contrast at the terraces. 
(a)

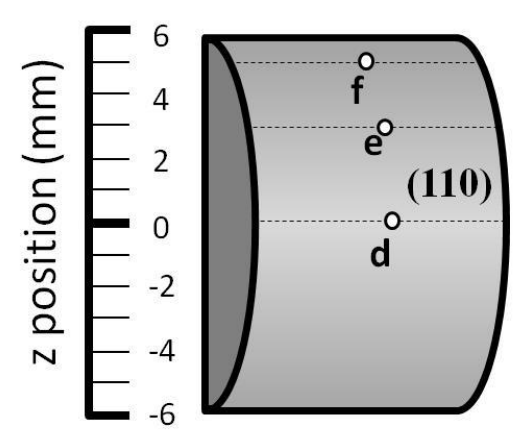

(b)

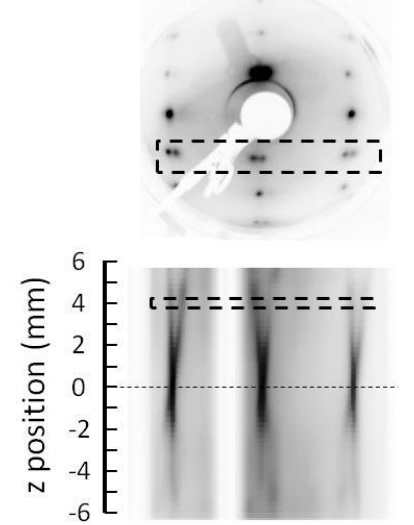

(d)

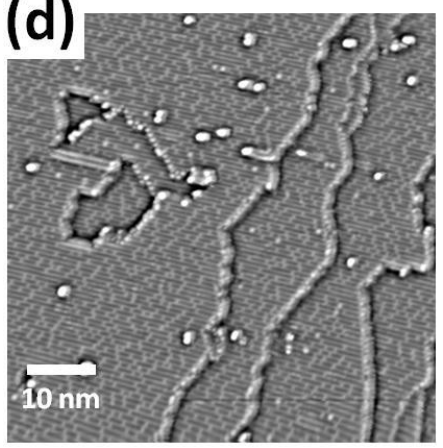

(g)

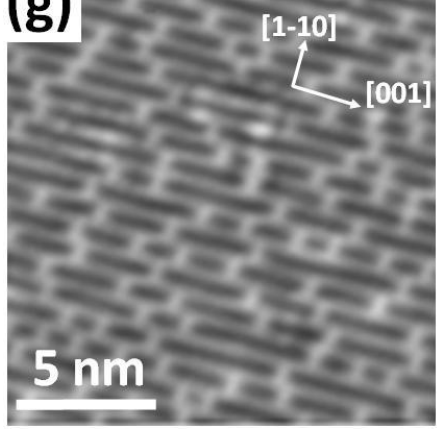

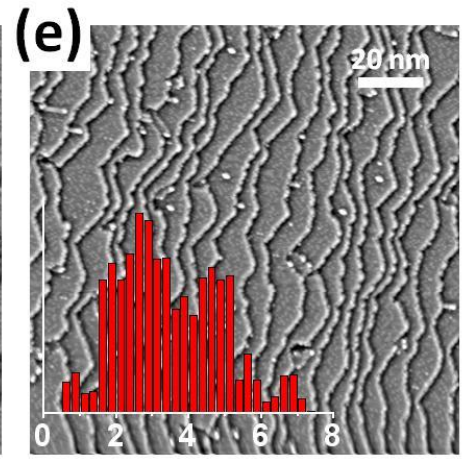

(h)

$\mathrm{O}_{\mathrm{br}} \mathrm{vac} \cdot \mathrm{Ti}_{4 \mathrm{f}}: \mathrm{Ti}_{5 \mathrm{f}}$

- $\mathrm{O}_{3 \mathrm{f}} \bullet \mathrm{O}_{\mathrm{br}} \bullet \mathrm{O}_{\mathrm{s}}$

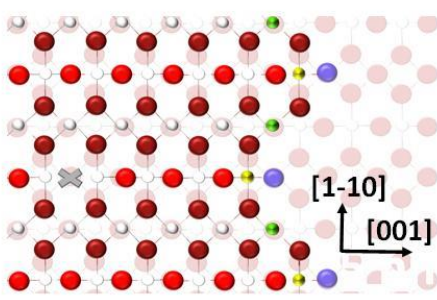

(c)
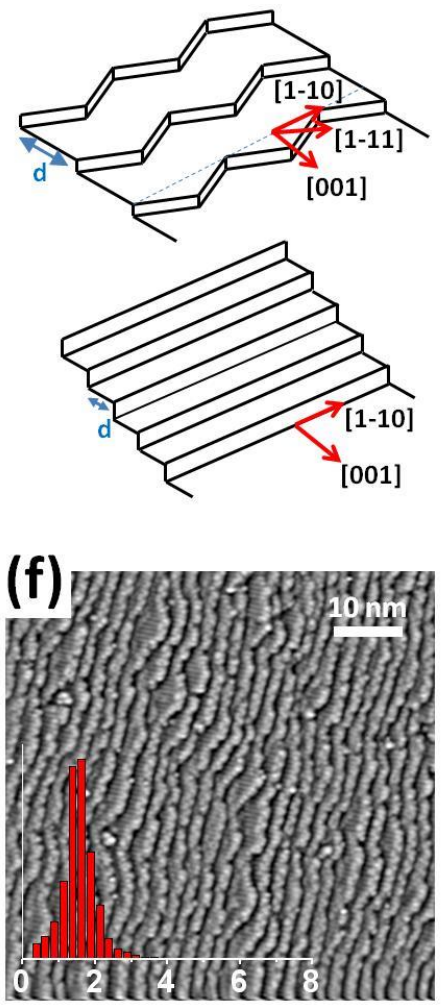

(i)

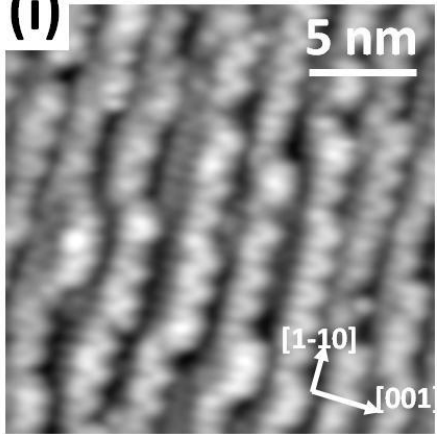

Figure 1. STM analysis across the c- $\mathrm{TiO}_{2}(110)$ surface. (a) Schematic drawing of the $c$-TiO $(110)$ sample. The (110) plane is located at the center of the curved crystal, and the step density smoothly increases towards both sides. Marked are the positions at which STM images $(d)-(f)$ were obtained. (b) Top, LEED pattern (electron energy $45 \mathrm{eV}$ ) acquired at $z=4 \mathrm{~mm}$ (referring to the scale in (a)), with the spot splitting characteristic of step arrays. Bottom, LEED image created by individual line scans (indicated by the dotted box in the top panel) across the curved crystal. (c) Sketch of the observed step structures in STM, namely alternating [1-11] segments, abundant at position e, and straight [1-10] steps, dominating at location $f$. (d-f) Selected STM images at the positions indicated in panel $(a)$. For $(e)$ and $(f)$ the terrace width histograms are included. $(\mathrm{g})$ Zoom-in image of a (110) terrace with $O_{b r}$ vac's appearing as bright, short features connecting bright Ti rows. (h) Atomic model for the [1-10] step, from Ref. ${ }^{12}$, with two-fold coordinated $O_{s}$ atoms highlighted in blue. (i) Zoom-in of $(f)$ showing narrow, $O_{b r}$ vac's-free terraces, regularly separated by [1-10] steps that feature triangularly-shaped $S_{t}$ protrusions (one $S_{t}$ triangle corresponds to an armchair period, namely to one pair of $O_{s}$ ). 
The smoothly varying step density $1 / d$ of the $\mathrm{c}-\mathrm{TiO}_{2}(110)$ sample is directly visualized in a LEED “ $z$-scan", shown in Fig. 1 (b). In such a $z$-scan the sample is displaced vertically ( $z$-scale in Fig. 1) in front of the $0.3 \mathrm{~mm}$ broad electron beam. The example on top of Fig. 1 (b) shows the pattern taken at $z=4 \mathrm{~mm}\left(\alpha=8^{\circ}\right)$; it exhibits the spot-splitting characteristic for stepped surfaces $^{19}$. The dotted box indicates a selected line over this LEED pattern, crossing the (-1-1), $(-10)$ and (-11) spots. The z-scan in the bottom of Fig. 1 (b) is built with individual spot line profiles taken in $\Delta z=0.5 \mathrm{~mm}$-steps across the surface. It shows a linear variation of the spot splitting at both sides of the crystal, reflecting a linear change in $1 / d$.

Despite the extensive sputter-annealing, the curved crystal surface remained stable, and continued to exhibit a position-dependent terrace-step structure. The STM images in panels (d)(f) correspond to the strongly reduced surface and are taken at the three characteristic positions marked in Fig. 1 (a). At the (110)-oriented center of the crystal the striped pattern reflects the presence of the alternating rows of bridge-bonded $\mathrm{O}$ and $\mathrm{Ti}$ atoms, better seen in the zoomed area of Fig. $1(\mathrm{~g})$. The $\mathrm{O}_{\mathrm{br}}$ vac's appear as bright features bridging Ti rows in STM images of $\mathrm{r}$ $\mathrm{TiO}_{2}(110)^{5,20}$. The morphology of step edges varies across the c- $\mathrm{TiO}_{2}$ sample in the way qualitatively sketched in Fig. 1 (c). At relatively low step densities [Fig. 1 (e)], zig-zag edges are observed with long segments parallel to [1-11] and [-111]. Such [1-11]-type steps are energetically favorable in rutile $\mathrm{TiO}_{2}(110)$, as compared to [1-10] steps ${ }^{1,11,14}$. At large step densities (1/d), the zig-zagging is reduced and steps mostly align parallel to the [1-10] direction, as shown in Figs. 1 (f) and (i). In all cases, step edges exhibit triangularly-shaped protrusions ( $S_{t}$ protrusions) at positive STM bias. At [1-11] steps, bright features have been previously associated with vacancies $\left(\mathrm{O}_{\mathrm{s}} \mathrm{vac}^{\prime} \mathrm{s}\right)^{13}$. In contrast, $\mathrm{S}_{\mathrm{t}}$ protrusions at [1-10] steps have been linked to the twofold-coordinated bridge oxygen atoms $\left(\mathrm{O}_{\mathrm{s}}\right)$ that terminate the armchair edges ${ }^{12}$, see the sketch in Fig. 1(h). Although the oxygen coordination is twofold also in terraces, here the $\mathrm{O}_{\mathrm{s}}$ atoms are bound to under-coordinated Ti atoms, which results in a larger contribution to the empty states image in STM (see Figure S9 in the Supporting Information file), as compared to $\mathrm{O}_{\mathrm{br}}$ atoms in terraces. Since the density of $\mathrm{O}_{\mathrm{s}}$ sites is selectively tuned across the curved surface, an important question is whether such $\mathrm{O}_{\mathrm{s}}$ atoms are active sites at the $\mathrm{TiO}_{2}(110)$ surface, i.e., whether they exhibit enhanced chemical affinity to adsorbates, and whether and how they contribute to the doping charge of the crystal.

By sputter-annealing we can transform the stoichiometric to the reduced stage of the crystal which results in a change in the number of $\mathrm{O}_{\mathrm{br}} v \mathrm{vac}^{\prime} \mathrm{s}$, as illustrated in Figs. 2 (a) and (b). In contrast, the reduction process has a smaller effect on the density of triangularly-shaped protrusions, which are already visible at the slightly reduced sample [Fig. 2 (a)]. $\mathrm{O}_{\mathrm{br}}$ vacancies 
have been reported to migrate from terraces to steps, leading to $\mathrm{O}_{\mathrm{br}}$ vac's-depleted terrace fringes in the proximity of [1-11]-oriented steps ${ }^{13}$. Such $\mathrm{O}_{\mathrm{br}}$ vac's migration effect supports our results displayed in Fig. 2. Here we statistically evaluate the distance from the step edge to the nearest $\mathrm{O}_{\mathrm{br}}$ vacancy in $\sim 10$ STM images of the reduced surface. Assuming that both step edges are equivalent, the peak at $\sim 1.6 \mathrm{~nm}$ suggests that the density of $\mathrm{O}_{\mathrm{br}}$ vac's will be very low in terraces with a width below $d_{\min }=2 \times 1.6=3.2 \mathrm{~nm}$. Finally, oxygen vacancies of terraces and $\mathrm{S}_{\mathrm{t}}$ features are tested against water adsorption in Fig. 2 (d). Water molecules dissociate in an analogous way at $\mathrm{O}_{\mathrm{br}}$ vac's of terraces and [1-10] steps, suggesting their similarly reactive nature.

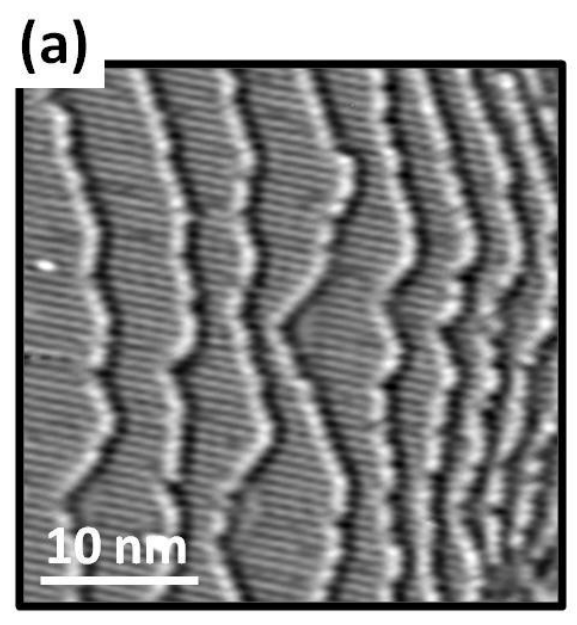

(c)

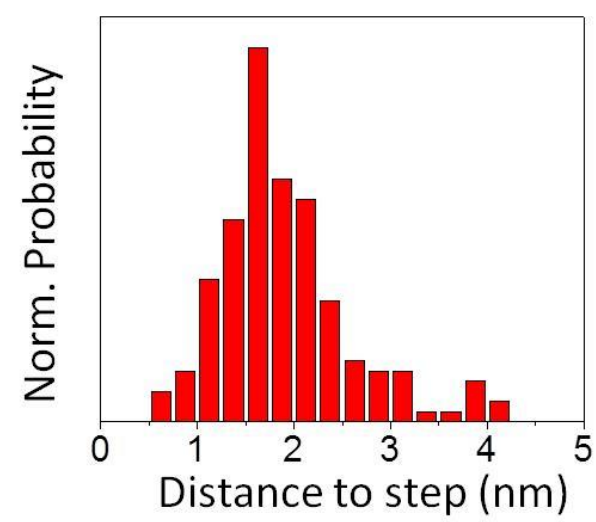

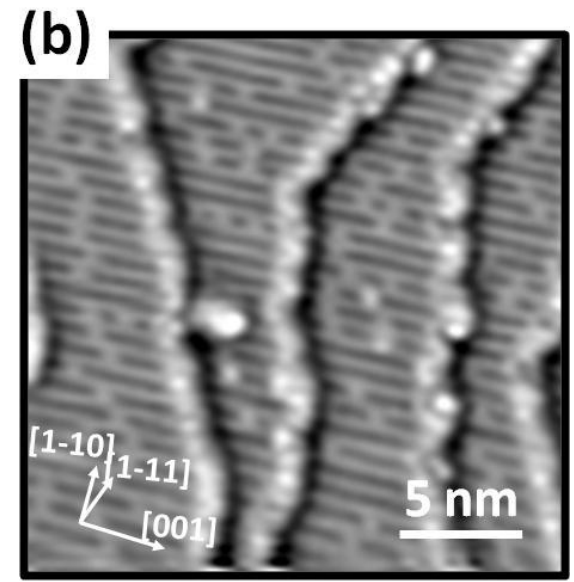

(d)

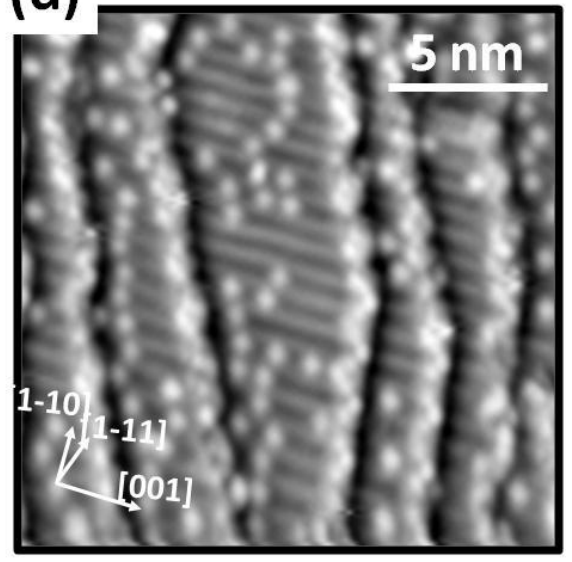

Figure 2. Active sites at c-TiO ${ }_{2}$. STM images with a similar step density for (a) the slightly reduced and (b) the strongly reduced surface. In (a), $O_{b r}$ vacancies are not observed and $S_{t}$ triangular protrusions already decorate step edges. In (b) we observe 1-2 $O_{b r}$ vacancies per Ti row in variable $3-8 \mathrm{~nm}$ wide terraces. (c) Analysis of the average distance from the step to the closest $O_{b r}$ vacancy, made over a set of STM images with 3 - $8 \mathrm{~nm}$ terrace width on the strongly reduced surface. (d) Stepped area of the strongly reduced surface after 0.1 Langmuir $\mathrm{H}_{2} \mathrm{O}$ adsorption. 
A statistical analysis of the terrace size in step arrays performed across the curved crystal provides key insights into the interplay between steps and vacancies on stepped rutile $\mathrm{TiO}_{2}(110)$. This analysis delivers probability histograms for the local terrace width $d$, such as those shown in the insets of Figs. 1 (e) and (f). Note that the histogram of Fig. 1 (e) (strongly reduced surface) shows a maximum probability at around $d \sim 2.5 \mathrm{~nm}$. This is significantly narrower than the mean terrace width $\widetilde{d}$ at that sample position. This observation suggests that steps tend to group, forming bunches with a critical spacing $d_{c}$. It is straightforward to deduce such a step-bunching effect in the intensity plot of Fig. 3 (a). Here the image is built with all terrace width distribution histograms taken across the curved surface, and plotted against the corresponding average terrace width $\widetilde{d}$ (vertical axis). The thick $\widetilde{d}=d$ line would mark the maximum for a symmetric histogram, i.e., the probability maximum expected for a simple, periodic step superlattice ${ }^{21}$. In Fig. 3 (a) we observe that for step arrays with average terrace width up to $\widetilde{d} \sim 3 \mathrm{~nm}$ the histogram maximum lies on the $\widetilde{d}=d$ line. In contrast, for arrays with larger $\widetilde{d}$ the maximum deviates from the $\widetilde{d}=d$ line, at a constant $d_{c}=2.8 \mathrm{~nm}$ (dotted line). Note that $d_{c}$ falls right below the minimum $d_{\min }=3.2 \mathrm{~nm}$ width that allows $\mathrm{O}_{\mathrm{br}} \mathrm{vac}$ 's within terraces. Therefore, for $\widetilde{d}>d_{c}$, the stepped surface exhibits both $\mathrm{O}_{\mathrm{br}}$ vac's-filled $\left(d>d_{c}\right)$ terraces and $\mathrm{O}_{\mathrm{br}}$ vac's-free $\left(d<d_{c}\right)$ bunches.

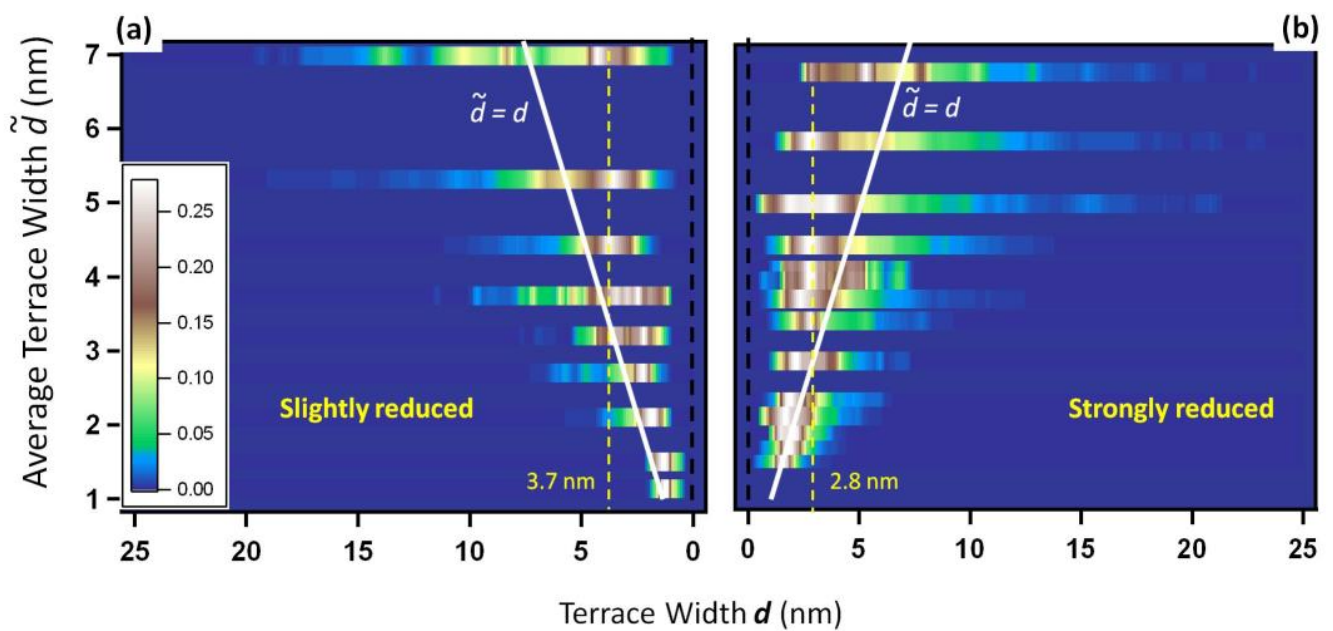

Figure 3. Terrace width distribution analysis for $\mathbf{c}^{-\mathrm{TiO}_{2}}$. Terrace width distributions plotted as a function of the mean terrace width $\widetilde{d}$ for (a) the slightly reduced and (b) the strongly reduced curved crystal. The white line marks the maximum in a Gaussian-like histogram. A step-bunching phase (dashed line) segregates at $d_{c}=3.7 \mathrm{~nm}$ in (a) and $d_{c}=2.8 \mathrm{~nm}$ in (b), while the histogram becomes more asymmetric for large $\widetilde{d}$ values. $d_{c}$ falls right below the observed limit $d_{\text {min }}$ in Fig. 2 (c).

The equilibrium shape of the step lattice results from the interplay of different interactions, mostly of elastic origin, but also of other nature, such as entropic, electrostatic, and electronic ${ }^{21}$. Steps lead to local crystal lattice relaxations, which extend several nanometers in all directions. 
Elastic fields of contiguous steps overlap, leading to repulsive, $\propto 1 / d^{2}$ interactions. When no other interactions exist, step-step repulsion results in periodic step arrays. Step bunching reveals the presence of other contributions to the energy balance. This is the case of faceting induced by surface reconstructions, such as the herring-bone in $\mathrm{Au}(111)^{22}$ and the $7 \times 7$ in $\mathrm{Si}(111)^{23}$, or by surface charge neutrality in $\mathrm{ZnO}^{24,25}$, as well as the bunching triggered by electric-dipole-like attractive interactions between steps in $\mathrm{Cu}(100)^{26}$ or $\mathrm{GaAs}(001)^{27}$. In the stepped $\mathrm{TiO}_{2}(110)$ system analyzed here, the structural distortions caused by $\mathrm{O}_{\mathrm{br}} \mathrm{vac}^{\prime} \mathrm{s}$ necessarily alter the surface free energy at (110) terraces. The existence of the stable, $\mathrm{O}_{\mathrm{br}}$ vac'sfree stepped phase suggests that $\mathrm{O}_{\mathrm{br}}$ vac's increase such elastic energy, such that a $\mathrm{O}_{b r}$ vac's-free phase with $d_{c} \sim 2.8 \mathrm{~nm}$ is promoted. However, the slightly reduced surface, with a much lower density of $\mathrm{O}_{\text {br }}$ vac's, also exhibits step bunching [Fig. 3 (a)], although with slightly wider critical terrace $d_{c} \sim 3.7 \mathrm{~nm}$. In the absence of $\mathrm{O}_{\mathrm{br}} \mathrm{vac}^{\prime} \mathrm{s}$ at terraces, or any other kind of structural defects or reconstructions, a step bunching phase is explained by attractive interactions between steps. Therefore, a more complex scenario appears to be present, where both attractive step-stepinteractions and $\mathrm{O}_{\mathrm{br}}$ vacancies enter the elastic energy balance, leading to step bunching at $d_{c}$ values that depend on the density of $\mathrm{O}_{\mathrm{br}}$ vacancies.

Terrace-width histogram plots like these of Fig. 3 contain further information. As recently shown for a c-Pt(111) $\mathrm{crystal}^{28}$, they allow visualizing the universal transition from entropic to elastic interactions in step arrays ${ }^{21}$. In fact, at high step densities strong repulsive $1 / d^{2}$ interactions define straight edges, but at low densities, steps do not interact with each other so effectively, and the step energy dictates the edge morphology. Non-interacting steps are allowed to twist, exhibiting, e.g., the favorable [1-11]-like orientations forming a zig-zag in c- $\mathrm{TiO}_{2}(110)$, or a round profile in c- $\mathrm{Pt}(111)$, which reflects minor energy variations among distinct facet orientations in metals. In either case, $\mathrm{c}-\mathrm{TiO}_{2}(110)$ or $\mathrm{c}-\mathrm{Pt}(111)$, the terrace size distributions are rather symmetric, Gaussian-like in dense step lattices (interacting steps), but develop a larger asymmetry, or tail towards big $d$ values, in sparse lattices. This tail is clearly visible in both plots of Fig. 3. It reflects that there is a large number of step atom configurations of similar energy, rendering a large entropic contribution to the surface free energy ${ }^{21}$.

The doping effect caused by steps featured with $S_{t}$ triangular protrusions and $\mathrm{O}_{\mathrm{br}}$ vac's is tested through the Ti $3 \mathrm{~d}$ gap state, which is the spectroscopic signature for all electron-donating defects in $\mathrm{TiO}_{2}{ }^{1,3,4,29}$. ARPES spectra are analysed as a function of the step density ( $z$-scan, as in Fig. 1 (b)) and the reduction level (see Fig. 4). To clearly separate $\mathrm{O}_{\mathrm{br}}$ and step contributions, we focus on the slightly reduced surface, where the presence of oxygen defects is lowest: For the more stepped part of the sample the surface is practically free of $\mathrm{O}_{\mathrm{br}} \mathrm{vac}$ 's, whereas the 
center has an irrelevant contribution of steps. Panel Fig. 4 (a) corresponds to a $z$-scan of the Ti $3 \mathrm{~d}$ gap state from the center (top) to the edge (bottom) of the lightly reduced sample, under normal emission conditions $\left(\theta=0^{\circ}\right)$. The persistence of the Ti $3 \mathrm{~d}$ gap state is consistent with the fact that both $\mathrm{O}_{\mathrm{br}} \mathrm{vac}$ 's of terraces and steps are electron-donating entities. Yet an energy shift is observed in the gap state from the center to the edge of the crystal. Background-subtracted spectra taken at the center and the densely stepped region are directly overlaid (Fig. 4 (a) bottom). A clear $\Delta E=110 \pm 20 \mathrm{meV}$ shift to higher binding energies is found from the (110) plane $(\mathrm{E}=-0.82 \mathrm{eV})$ to the stepped surface $(\mathrm{E}=-0.93 \mathrm{eV})$. A close view at the corresponding $\mathrm{O} 2 \mathrm{p}$ evolution discards changes in band bending as a possible reason for the observed shift, as discussed in the $\mathrm{SI}^{16}$. An energy shift in the gap state peak of the (110) plane could be expected at distinct Ti environments, such as steps and defects, which imply different lattice distortion (polaron). It can also arise due to the different coordination of Ti atoms at the step edge, which leads to an effectively different valence state. For example, for the $2 \times 1$ phase with nominal $\mathrm{Ti}_{2} \mathrm{O}_{3}$ composition, i.e., lower Ti coordination to $\mathrm{O}$ atoms, a $120 \mathrm{meV}$ shift to higher binding energy has been reported ${ }^{29}$. 

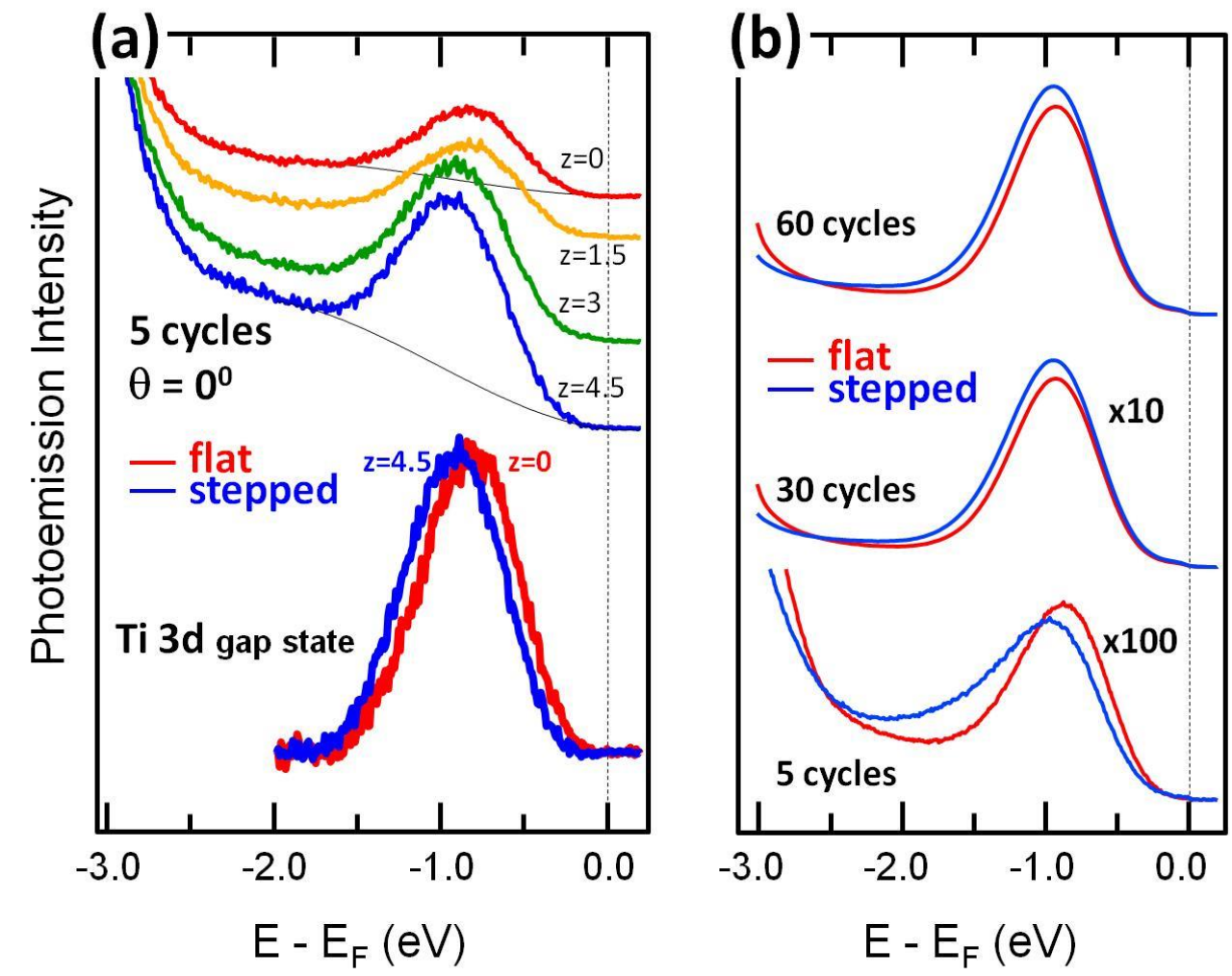

(c)

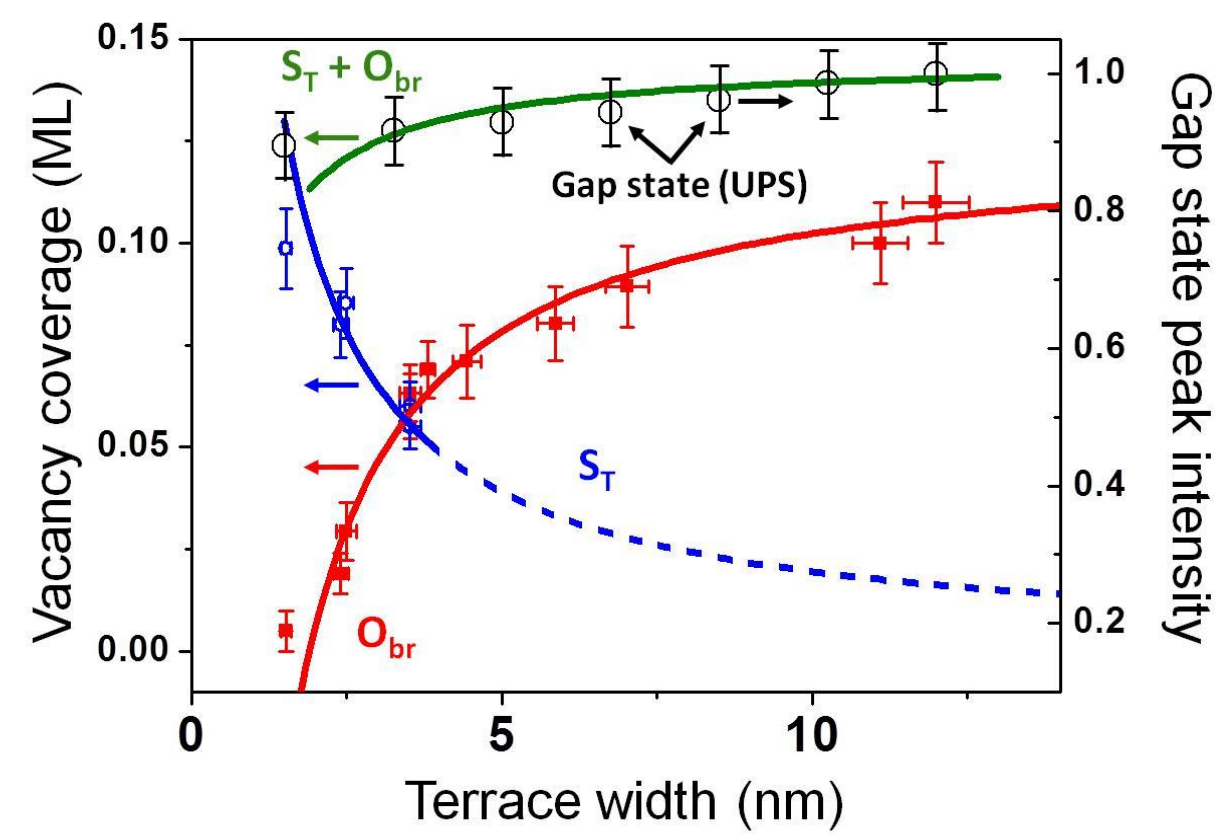

Figure 4 Doping effect studied by the modification of the Ti 3d gap state (a) z-scan of the gap state peak obtained at normal emission $\left(\theta=0^{\circ}\right)$ for the slightly reduced surface. The bottom shows normalized spectra taken at the $z=0 \mathrm{~mm}$ and $z=4.5 \mathrm{~mm}$ after background subtraction (thin lines). (b) Integrated photoemission spectra of the $\mathrm{c}-\mathrm{TiO}_{2}$ acquired at three different reduction stages (sputter-annealing cycles), at flat (red, $z=0 \mathrm{~mm}$ ) and stepped (blue, $z=5 \mathrm{~mm}$ ) positions of the crystal. (c) $O_{b r}$ vac's (red squares) and $S_{t}$ protrusions (blue dots) concentration, as determined from STM images of the strongly reduced surface. The empirical $O_{b r}(d)=1 / 1.9-1 / d$ and $1 / d$ lines fit the data. The black line represents the 
sum of $\mathrm{O}_{b r}$ and $S_{t}$ protrusions. It correlates with the almost constant intensity of the angle integrated gap state peak measured in the z-scan (white dots) on the same reduced surface.

To quantify the variation of the doping charge in the curved crystal we analyse the photoemission intensity of the $\mathrm{Ti} 3 \mathrm{~d}$ gap state. For this purpose, a wide emission angle integration is required, given the large angular variation of the gap state intensity in ARPES, as discussed in detail in the $\mathrm{SI}^{16}$. In Fig. 4 (b) the Ti 3d gap states for the curved crystal center $(z=0)$ and the highly stepped edge $(z=5 \mathrm{~mm})$ [points $\boldsymbol{d}$ and $\boldsymbol{f}$ in Fig. 1 (a), respectively] are compared, at different degrees of surface reduction. Data correspond to the full integration of the emission plane accessible in the experiment, namely $\Delta \theta=-10^{\circ}$ to $+70^{\circ}$ in the [1-10] direction parallel to surface steps, and $\Delta \beta= \pm 12^{\circ}$ in the [00-1] perpendicular direction (cf. Fig. S6). The step-related energy-shift persists, but it is smaller at strongly reduced surfaces. The latter is consistent with the widely accepted picture that the polarons first occupy special sites (at the step edges in the present case), followed by regular lattice positions ${ }^{30}$.

The advantage of the curved surface is that the very same reduction process is applied to all surface orientations at once. Thus, it is remarkable to observe that the gap state shows minor intensity differences from flat to stepped surfaces at any reduction stage. This indicates that the total doping charge, originating from both, $\mathrm{O}_{\mathrm{br}} \mathrm{vac}^{\prime} \mathrm{s}$ and $\mathrm{S}_{\mathrm{t}}$ steps, remains constant across the curved surface. In Fig. 4 (c) we study the correlation between the concentration of $\mathrm{O}_{\mathrm{br}} \mathrm{vac}$ 's and $\mathrm{S}_{\mathrm{t}}$ triangles and the gap state intensity. Red and blue data points represent the density (in monolayers, ML) of $\mathrm{O}_{\mathrm{br}}$ and $\mathrm{S}_{\mathrm{t}}$ triangles, measured directly from STM images as a function of the terrace size $d$. White dots correspond to the gap state intensity (area under the peak), averaged to the photon intensity over selected ARPES z-scans of the reduced surface. Error bars reflect the intensity variation between stepped and non-stepped areas, which may vary by $10 \%$, depending on the emission angle integration $\left(\right.$ see $\mathrm{SI}^{16}$ ). The concentration of triangles follows the step density variation $1 / d$ (blue line), meaning that such features saturate step edges at any terrace width. The dotted portion of the line indicates that triangles cannot be resolved in large area images, which are needed to properly quantify STM data at low step densities. By contrast, the concentration of $\mathrm{O}_{\mathrm{br}} \mathrm{vac}^{\mathrm{s}} \mathrm{s}$ is fitted with the empirical function $\mathrm{O}_{\mathrm{br}}(d)=1 / D_{0}-1 / d$ (red line), which reflects in the most simple way the qualitative observations of Figs. 2 and 3, namely the existence of $\mathrm{O}_{\mathrm{br}}$ vac-free areas close to step edges (also $1 / d$ dependent) and a critical terrace size below which $\mathrm{O}_{\mathrm{br}}$ vac's vanish $\left(1 / D_{0}\right)$. The best fit is obtained for $D_{0}$ values around $2.0 \mathrm{~nm}$, i.e., slightly below the $d_{c}=2.8 \mathrm{~nm}$ value deduced from Fig. 3 .

The most interesting conclusion from Fig. 4 (c) is that the direct sum of the fitting lines for $\mathrm{O}_{\mathrm{br}}$ vac's and $\mathrm{S}_{\mathrm{t}}$ triangles leads to almost the complete cancellation of $1 / d$ dependence, and hence to 
a constant line that fits the photoemission data on top. This means that every $S_{t}$ protrusion at a step edge donates to the crystal the same electronic charge as one $\mathrm{O}_{\mathrm{br}}$ vacancy on a (110) terrace. The question that arises is which physical entity donates the doping charge at the step edge. We have theoretically analysed the role of steps as electron dopants and found out that neither the low-coordinated $\mathrm{Ti}$ atoms nor the $\mathrm{O}_{\mathrm{s}}$ atoms at the step-edges introduce any additional states inside the gap, i.e., they do not donate any electrons to the crystal. We thus analysed the interplay between the two-fold oxygen vacancies and the step edges (see SI). We should notice that the presence of one $\mathrm{O}_{\mathrm{br}}$ vacancy per each $\mathrm{S}_{\mathrm{t}}$ triangle could directly explain the STM/ARPES results of Fig. 4. Interestingly, our calculations indicate that $\mathrm{O}_{\mathrm{br}}$ vacancies have lower formation energy at the upper corner of the armchair unit cell, as compared to $\mathrm{O}_{\mathrm{br}}$ vacancies in terraces. This suggests that, as observed in [1-11] steps, $\mathrm{O}_{\mathrm{br}}$ vacancies migrate to step edges, saturating them at a sufficient reduction stage (Fig. 4). On the other hand, as shown in Figure S9 of the SI file, the presence of $\mathrm{O}_{\mathrm{br}}$ vacancies at step edges does not considerably alter the STM image, due to the strong contribution from the $\mathrm{O}_{\mathrm{s}}$ atoms and the low coordinated Ti atoms at the step edge.

In summary, the curved-crystal approach allows us to rationally investigate the interplay between steps and $\mathrm{O}_{\mathrm{br}}$ vacancies in stepped rutile $\mathrm{TiO}_{2}$. Such crystals are remarkable stable; the step-terrace structures survives typical sputter-anneal cleaning cycles and even heating at atmospheric pressures (see SI). The STM analysis shows a smooth evolution from flat surfaces with abundant bridge-bonded $\mathrm{O}$ vacancies to densely stepped surfaces, featuring triangular stepedge protrusions. A step-bunching phase made of vacancy-free terraces is observed to segregate at relatively sparse step arrays, suggesting that $\mathrm{O}$ vacancies in stepped $\mathrm{r}-\mathrm{TiO}_{2}(110)$ interfere with the elastic/entropic step interaction, characteristic of vicinal surfaces. The photoemission analysis proves a transition from $\mathrm{O}_{\mathrm{br}}$ vacancy to step-edge doping, with different polaronic energies, but similar effect in the doping charge of the $\mathrm{TiO}_{2}$ crystal.

We acknowledge financial support from the Spanish Ministry of Economy (grant MAT201346593-C6-4-P and MAT2013-46593-C6-2-P) and the Basque Government (grant IT621-13 and IT756-13). MS and UD acknowledge support from the ERC Advanced Grant "OxideSurfaces". DSP and MM acknowledge support from the Marie Curie ITN “THINFACE”. 
1. Diebold, U. Surface Science Reports 2003, 48, (5-8), 53-229.

2. Wendt, S.; Sprunger, P. T.; Lira, E.; Madsen, G. K. H.; Li, Z.; Hansen, J. Ø.; Matthiesen, J.; Blekinge-Rasmussen, A.; Lægsgaard, E.; Hammer, B.; Besenbacher, F. Science 2008, 320, (5884), 1755-1759.

3. Papageorgiou, A. C.; Beglitis, N. S.; Pang, C. L.; Teobaldi, G.; Cabailh, G.; Chen, Q.; Fisher, A. J.; Hofer, W. A.; Thornton, G. Proceedings of the National Academy of Sciences 2010, 107, (6), 2391-2396.

4. Krüger, P.; Jupille, J.; Bourgeois, S.; Domenichini, B.; Verdini, A.; Floreano, L.; Morgante, A. Physical Review Letters 2012, 108, (12), 126803.

5. Setvin, M.; Franchini, C.; Hao, X.; Schmid, M.; Janotti, A.; Kaltak, M.; Van de Walle, C. G.; Kresse, G.; Diebold, U. Physical Review Letters 2014, 113, (8), 086402.

6. Kowalski, P. M.; Camellone, M. F.; Nair, N. N.; Meyer, B.; Marx, D. Physical Review Letters 2010, 105, (14), 146405.

7. $\quad$ Krüger, P.; Bourgeois, S.; Domenichini, B.; Magnan, H.; Chandesris, D.; Le Fèvre, P.; Flank, A. M.; Jupille, J.; Floreano, L.; Cossaro, A.; Verdini, A.; Morgante, A. Physical Review Letters 2008, 100, (5), 055501.

8. Deak, P.; Aradi, B.; Frauenheim, T. Phys. Rev. B 2012, 86, 195206.

9. Janotti, A.; Franchini, C.; Varley, J. B.; Kresse, G.; Valle, C. G. V. d. Phys. Stat. Sol. 2013, 7, 199-203.

10. Austin, I. G.; Mott, N. F. Adv. Phys. 2001, 50, 757-812.

11. Hardcastle, T. P.; Seabourne, C. R.; Brydson, R. M. D.; Livi, K. J. T.; Scott, A. J. The Journal of Physical Chemistry C 2013, 117, (45), 23766-23780.

12. Luttrell, T.; Li, W.-K.; Gong, X.-Q.; Batzill, M. Physical Review Letters 2009, 102, (16), 166103.

13. Martinez, U.; Hansen, J. Ø.; Lira, E.; Kristoffersen, H. H.; Huo, P.; Bechstein, R.; Lægsgaard, E.; Besenbacher, F.; Hammer, B.; Wendt, S. Physical Review Letters 2012, 109, (15), 155501.

14. Stausholm-Møller, J.; Kristoffersen, H. H.; Martinez, U.; Hammer, B. The Journal of Chemical Physics 2013, 139, (23), 234704.

15. Takahashi, H.; Watanabe, R.; Miyauchi, Y.; Mizutani, G. The Journal of Chemical Physics 2011, 134, (15), 154704.

16. Supplementary information file is provided at XXXX.

17. Li, M.; Hebenstreit, W.; Diebold, U.; Tyryshkin, A. M.; Bowman, M. K.; Dunham, G. G.; Henderson, M. A. The Journal of Physical Chemistry B 2000, 104, (20), 4944-4950.

18. Park, K. T.; Pan, M. H.; Meunier, V.; Plummer, E. W. Physical Review Letters 2006, 96, (22), 226105.

19. The spot-splitting depends on the electron energy in LEED, but, due to the finite probing depth, in-phase and out-of-phase interference conditions occur at different energies for different diffracted beams. This explains the absence of splitting in some of the spots in Fig. 1.

20. Diebold, U.; Anderson, J. F.; Ng, K.-O.; Vanderbilt, D. Physical Review Letters 1996, 77, (7), 1322-1325.

21. Jeong, H.-C.; Williams, E. D. Surface Science Reports 1999, 34, (6-8), 171-294.

22. Corso, M.; Schiller, F.; Fernández, L.; Cordón, J.; Ortega, J. E. Journal of Physics: Condensed Matter 2009, 21, (35), 353001.

23. Kopciuszyński, M.; Dyniec, P.; Zdyb, R.; Jałochowski, M. Physical Review B 2015, 91, (23), 235420.

24. Dulub, O.; Diebold, U.; Kresse, G. Physical Review Letters 2003, 90, (1), 016102. 
25. Zheng, H.; Gruyters, M.; Pehlke, E.; Berndt, R. Physical Review Letters 2013, 111, (8), 086101.

26. Frohn, J.; Giesen, M.; Poensgen, M.; Wolf, J. F.; Ibach, H. Physical Review Letters 1991, 67, (25), 3543-3546.

27. Magri, R.; Gupta, S. K.; Rosini, M. Physical Review B 2014, 90, (11), 115314.

28. Walter, A. L.; Schiller, F.; Corso, M.; Merte, L. R.; Bertram, F.; Lobo-Checa, J.; Shipilin, M.; Gustafson, J.; Lundgren, E.; Brion-Rios, A. X.; Cabrera-Sanfelix, P.; Sanchez-Portal, D.; Ortega, J. E. Nat Commun 2015, 6.

29. Sánchez-Sánchez, C.; Garnier, M. G.; Aebi, P.; Blanco-Rey, M.; de Andres, P. L.; MartínGago, J. A.; López, M. F. Surface Science 2013, 608, 92-96.

30. J.Robertson. Rep. Prog. Phys. 2006, 69, (2), 327-396. 